\title{
Update on C3 Glomerulopathy: A Complement-Mediated Disease
}

\author{
Fernando Caravaca-Fontán ${ }^{\mathrm{a}, \mathrm{b}}$ Laura Lucientes ${ }^{\mathrm{a}, \mathrm{c}}$ Teresa Cavero $^{\mathrm{d}}$ \\ Manuel Praga ${ }^{a, b}$ \\ anstituto de Investigación Hospital 12 de octubre (i+12), Madrid, Spain; bepartment of Medicine, Universidad \\ Complutense de Madrid, Madrid, Spain; 'Department of Immunology, Universidad Complutense de Madrid, Madrid, \\ Spain; ${ }^{d}$ Department of Nephrology, Hospital Universitario 12 de Octubre, Madrid, Spain
}

\section{Keywords}

Alternative complement pathway · C3 glomerulonephritis . Dense deposit disease - Mycophenolate mofetil

\begin{abstract}
C3 glomerulopathy (C3G) is a clinicopathologic entity secondary to dysregulation of the alternative complement pathway in plasma and the glomerular microenvironment. The current consensus definition of C3G relies on immunofluorescence staining criteria. However, due to its high clinical variability, these criteria may not be accurate enough in some clinical scenarios. Thus, a new pathogenic classification based on a cluster analysis of clinical, histologic, and genetic data has recently been proposed, which could also help identify patients at higher risk of progression. Several pathogenic abnormalities in complement genes have been described, and the role of autoantibodies in the disease is increasingly recognized, but still the genotype-phenotype correlations in C3G are poorly understood. C3G may be diagnosed in both children and adults. The spectrum of clinical manifestations is wide, although one of the most common clinical presentations is proteinuria with relatively preserved kidney function. In order to standardize the evaluation of kidney biopsies from these patients, a histopathologic index was recently proposed, including both parameters of activity and chronicity. However, this index has not yet been val-
\end{abstract}

idated in independent cohorts. Currently, no targeted therapies are available in clinical settings for the treatment of C3G, although several new molecules are under investigation. Treatment with corticosteroids plus mycophenolate mofetil has been shown to be associated with improved renal outcomes, as compared to other immunosuppressive regimens. Yet, the main determinants of treatment response with this regimen and the influence of the underlying pathogenic drivers have not been extensively studied. The therapeutic response to eculizumab, an anti-C5 monoclonal antibody, has been shown to be highly heterogeneous. Thus, its current clinical indication in C3G is restricted to rapidly progressive forms of the disease. To summarize, in recent years, several important advances have taken place in the understanding of C3G, but still further studies are warranted to elucidate the best therapeutic strategies that could improve prognosis of this entity.

(c) 2020 S. Karger AG, Basel

\section{Introduction, Definition, and Classification}

The complement system is a major component of the innate immune system [1] and has the ability to discriminate between the host and pathogens, for their elimination [2]. There are three main pathways of complement activation - the classical, lectin, and alternative pathways,

$\begin{aligned} & \text { karger@karger.com } \\ & \text { www.karger.com/nef }\end{aligned}$
Karger ${ }^{\prime /}$

Dr. Fernando Caravaca-Fontán

Instituto de Investigación Hospital 12 de Octubre (i+12)

Ave. Córdoba $\mathrm{s} / \mathrm{n}$

ES-28041 Madrid (Spain)

fcaravacaf@gmail.com 
Fig. 1. Histologic subtypes of C3G: C3GN, in which deposits may be located in the mesangium and along the subendothelial side of the GBM; DDD, characterized by the presence of highly electron-dense deposits within the GBM. Blue molecules represent C3 and its cleavage products. C3G, C3 glomerulopathy; DDD, dense deposit disease; GBM, glomerular basement membrane; C3GN, C3 glomerulonephritis.

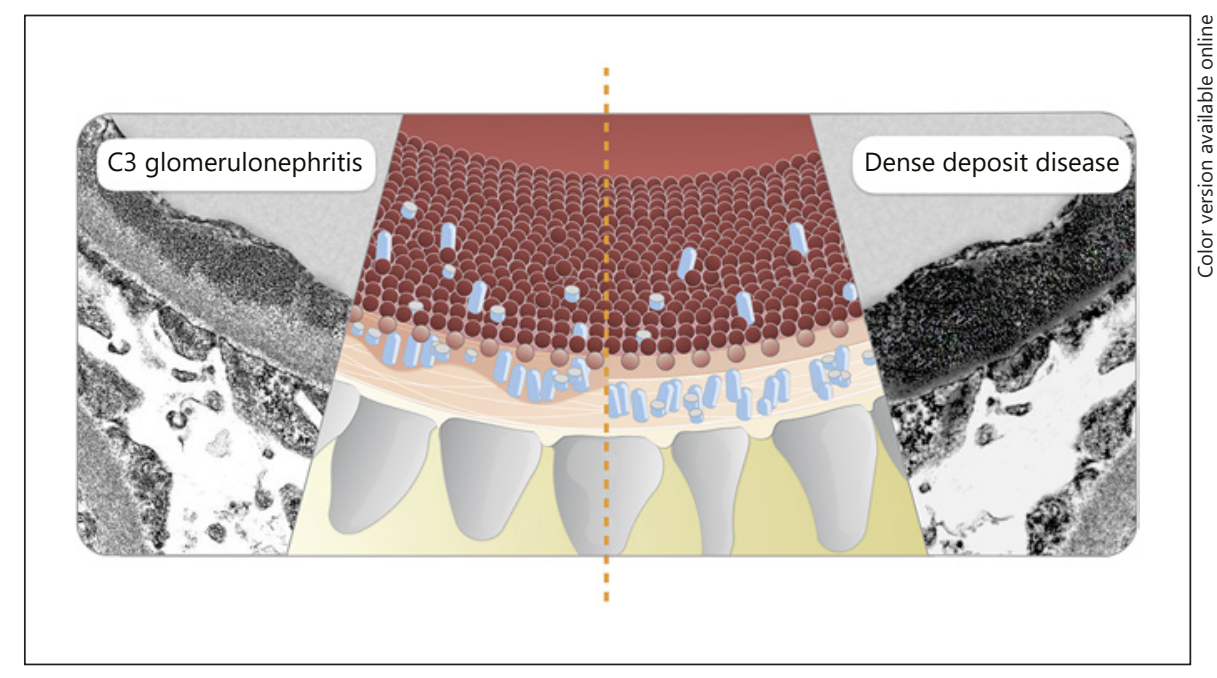

each leading to a common terminal pathway and the formation of the membrane attack complex [3]. The classical pathway is activated when the $\mathrm{C} 1$ complex binds to immune-complexes containing IgM or IgG. C1s, a subcomponent of $\mathrm{C} 1$, cleaves $\mathrm{C} 4$ and $\mathrm{C} 2$ and results in the assembly of C4b2a (classical pathway C3 convertase) [3]. The lectin pathway resembles the latter, although it is initiated when mannose-binding lectin binds to carbohydrates on the surface of pathogens [3].

The alternative complement pathway is persistently active at a low level [4], and it is initiated by the spontaneous hydrolysis of a thioester bond of the C3 molecule [3]. Under physiologic conditions, it is highly regulated by several plasmatic and membrane-bound molecules to avoid damage to self-tissues [1]. In recent years, dysregulation of the complement system has been involved in a myriad of kidney diseases, and $\mathrm{C} 3$ glomerulopathy $(\mathrm{C} 3 \mathrm{G})$ and atypical hemolytic uremic syndrome represent the two main complement-driven glomerular diseases [5].

C3G is a rare and heterogeneous clinicopathologic entity, secondary to the dysregulation of the alternative complement pathway in the fluid phase and the glomerular microenvironment $[4,6]$. This hyperactivation of the alternative complement pathway results in the deposition of $\mathrm{C} 3$ and its cleavage products within the glomeruli [6-8], leading to inflammation and progressive kidney disease. The estimated incidence of the disease is $1-3$ cases per million per year $[9,10]$, and it is more frequently diagnosed in childhood and adolescence, although older people can also be affected [11].

There are 2 major subtypes of C3G: dense deposit disease (DDD) characterized by the presence of highly elec- tron-dense deposits within the glomerular basement membrane (GBM), and C3 glomerulonephritis (C3GN), in which deposits may be located in the mesangium and along the subendothelial side of the GBM [4] (Fig. 1). However, due to the frequent overlapping features between these subtypes, some authors consider that they may all be part of the same disease spectrum [6].

The current consensus definition of $\mathrm{C} 3 \mathrm{G}$ relies on the presence of a positive glomerular immunofluorescence staining for C3, either isolated or at least two orders of magnitude greater than the rest of the immune reactants [12]. However, the cumulative experience has shown that these criteria could give rise to confusion in some clinical scenarios for several reasons [13, 14]. First, sometimes the results of immunofluorescence are borderline, and a subsequent kidney biopsy may reveal findings consistent with an immune-complex disease or vice versa $[13,14]$. Second, complement genetic abnormalities and/or autoantibodies against complement components can also be found in cases of immune-complex membranoproliferative GN (MPGN) [15]. Third, the immunofluorescence routinely used in clinical practice to stain for $\mathrm{C} 3$ stains for the fragment C3c, whereas in $\mathrm{C} 3 \mathrm{G}$, the predominant cleavage product that accumulates is C3dg [8].

Therefore, a new pathogenic classification of both immune-complex MPGN and C3G was proposed based on a cluster analysis of clinical, histologic, and genetic data from a large cohort of patients [14]. According to this classification, four different clusters are identified. Patients in clusters 1-3 have predominant fluid-phase complement activation, often present with low $\mathrm{C} 3$ levels, and have a high prevalence of complement genetic abnormal- 
Table 1. Genomic rearrangements associated with C3G (Adapted from Smith et al. [5])

\begin{tabular}{|c|c|c|c|}
\hline \multirow[t]{2}{*}{ FHR2 $_{1,2}-$ FHR5 $_{1-9}$} & $4: 5: 6: 7: 8: 9$ & DDD & $\begin{array}{l}\text { Chen Q, Wiesener M, Eberhardt HU, et al. Complement factor } \\
\text { H-related hybrid protein deregulates complement in dense deposit } \\
\text { disease. J Clin Invest. 2014;124:145-55 }\end{array}$ \\
\hline & & C3GN & $\begin{array}{l}\text { Veszeli N, Garam N, Mezö B, et al. Comprehensive analysis of copy } \\
\text { number variations of complement factor H (CFH) and CFH-related } \\
\text { genes in patients with glomerulonephritis or atypical hemolytic uremic } \\
\text { syndrome. Mol Immunol. 2019;114:458 }\end{array}$ \\
\hline FHR $_{1,2}-$ FHR $_{1-5}$ & $1: 2: 1: 2: 3$ & C3GN & $\begin{array}{l}\text { Veszeli N, Garam N, Mező B, et al. Comprehensive analysis of copy } \\
\text { number variations of complement factor H (CFH) and CFH-related } \\
\text { genes in patients with glomerulonephritis or atypical hemolytic uremic } \\
\text { syndrome. Mol Immunol. 2019;114:458 } \\
\text { Malik TH, Lavin PJ, Goicoechea de Jorge E, et al. A hybrid CFHR3-1 } \\
\text { gene causes familial C3 glomerulopathy. J Am Soc Nephrol. } \\
\text { 2012;23:1155-60 }\end{array}$ \\
\hline FHR1 $_{1,2}-$ FHR5 $_{1-9}$ & $1: 2: 1: 2: 3: 4: 5: 6: 7: 8: 9$ & DDD/C3GN & $\begin{array}{l}\text { Togarsimalemath SK, Sethi SK, Duggal R, et al. A novel CFHR1-CFHR5 } \\
\text { hybrid leads to a familial dominant C3 glomerulopathy. Kidney Int. } \\
\text { 2017;92:876-87 }\end{array}$ \\
\hline FHR $_{1-4}-\mathrm{FHR}_{1-5}$ & $1: 2: 3: 4: 1: 2: 3: 4: 5$ & C3GN & $\begin{array}{l}\text { Tortajada A, Yébenes H, Abarrategui-Garrido C, et al. C3 } \\
\text { glomerulopathy-associated CFHR1 mutation alters FHR oligomerization } \\
\text { and complement regulation. J Clin Invest. 2013;123:2434-46 }\end{array}$ \\
\hline FHR5 $_{1,2}-$ FHR2 $2_{1-4}$ & $1: 2: 1: 2: 3: 4$ & C3GN & $\begin{array}{l}\text { Xiao X, Ghossein C, Tortajada A, et al. Familial C3 glomerulonephritis } \\
\text { caused by a novel CFHR5-CFHR2 fusion gene. Mol Immunol. } \\
\text { 2016;77:89-96 }\end{array}$ \\
\hline FHR $_{1,2}-$ FHR $_{1-5}$ & $1: 2: 1: 2: 3: 4: 5$ & C3GN & $\begin{array}{l}\text { Gutiérrez Tenorio J, Márquez Tirado B, Merinero H, et al. A novel C3G- } \\
\text { associated FHR-1 mutant protein provides insight into the molecular } \\
\text { basis of complement deregulation. Mol Immunol. 2019;114:487 }\end{array}$ \\
\hline
\end{tabular}

SCRs are represented as squares. Colors indicate the FHR protein implicated and the numbers indicate the SCR. FHR1, yellow (5 SCRs); FHR2, blue (4 SCRs); FHR3, green (5 SCRs); FHR5, red (9 SCRs). SCR, short consensus repeat; FHR, factor H-related; DDD, dense deposit disease; C3G, C3 glomerulopathy; C3GN, C3 glomerulonephritis.

ities; patients in cluster 4 have predominant complement activation on surfaces, have normal serum C3 levels, and are associated with worse renal outcomes [14].

This newly proposed classification could be more useful for clinicians because, as opposed to the pathologic classification, it addresses the underlying pathogenesis of the disease. However, this classification has not yet been validated in other cohorts and may also be subject to other limitations [14].

\section{Pathogenesis}

Alternative complement pathway dysregulation is the central event in the pathogenesis of the disease. This unrestrained complement activation may be driven by ge- netic abnormalities in complement genes [15-19], autoantibodies against complement components, or nephritic factors (NeFs) that stabilize $\mathrm{C} 3$ and/or $\mathrm{C} 5$ convertases [6, $20,21]$.

Complement genetic abnormalities may be found in about $25 \%$ of patients $[10,15,17,22]$, mostly affecting the $C 3$ gene, $C F B, C F H, C F I$, and $C F H R$, which encode complement component $\mathrm{C} 3$, factor $\mathrm{B}(\mathrm{FB})$, factor $\mathrm{H}(\mathrm{FH})$, factor I (FI), and FH-related (FHR) proteins, respectively [6, 18]. Most pathogenic variants in $C 3$ affect the recognition sites for the binding of $\mathrm{FH}$ and FI, leading to an improper cleavage of the molecule [23]. Pathogenic variants in $C F B$ may confer gain-of-function properties [18], whereas certain abnormalities in FI may reduce its levels and compromise the cofactor activity [17]. On the other hand, several pathogenic variants have been described in $\mathrm{CFH}$ 
(both in heterozygosity and homozygosity), mainly affecting the N-terminal region, which is critical for fluidphase complement regulation $[7,18]$.

Rare variants (minor allele frequency $<1 \%$ ) may also be involved in the pathogenesis of C3G. Recent studies have reported an accumulation of rare variants in C3G patients, as compared to controls, mainly in the candidate genes $C F H, C 3, C F B$, and $C F I[15,24]$. Genomic rearrangements in the $C F H$-CFHR locus may result in the generation of mutant proteins (hybrid proteins or proteins with internal duplications), with the ability to compete with FH on certain surfaces, such as the GBM $[6,18$, $25,26]$.

Several well-documented familial cases of C3G with underlying genomic rearrangements in the $\mathrm{FH}$ gene family have been described in the recent years. The majority of genomic rearrangements specifically associated with $\mathrm{C} 3 \mathrm{G}$ result in the duplication of the dimerization domains of FHR-1, FHR-2, and FHR-5 (Table 1). In fact, a novel C3G-associated FHR1 mutant protein has recently been reported, with duplication of the dimerization domains (short consensus repeat domains 1 and 2) and consequent interference in complement regulation [27].

In addition to the aforementioned genetic abnormalities in complement genes, there are also some diseaselinked polymorphisms in $C F H, C 3, C F B$, and $M C P$ that may influence the complement activity and susceptibility to inflammatory and infectious diseases. For instance, the $\mathrm{CFH}-\mathrm{H} 1$ haplotype has been found to confer higher risk for the development of $\mathrm{C} 3 \mathrm{G}$, whereas $\mathrm{CFH}-\mathrm{H} 2$ has been found to confer protection [28]. In addition, the extended haplotype $\mathrm{CFH}-\mathrm{H} 4$, which contributes to the deletion of the CFHR3 and CFHR1 genes, has also been identified as a protective factor for C3G [29]. Furthermore, other common polymorphisms in $C F H, M C P$, and $T H B D$ have been associated with an increased susceptibility to the disease [15].

The main autoantibodies associated with C3G are the so-called NeFs, which comprise a heterogenous group of antibodies against neoepitopes generated in $\mathrm{C} 3$ or $\mathrm{C} 5$ convertases with the capacity to stabilize the molecule and prolong its half-life [20]. Two major subtypes of C3 nephritic factors ( $\mathrm{C} 3 \mathrm{NeFs})$ have been described: properdin-dependent $\mathrm{C} 3 \mathrm{NeFs}$ responsible for the activation of C5 convertase, also called C5NeFs [30], and properdinindependent $\mathrm{C} 3 \mathrm{NeFs}$ that target the $\mathrm{C} 3$ convertase, as reflected by low $\mathrm{C} 3$ levels but normal serum levels of the terminal complement pathway [20]. C3NeFs (properdin-independent) have mainly been associated with

Update on C3 Glomerulopathy
DDD, whereas C5NeFs have been associated with C3GN [21].

In addition, $\mathrm{NeFs}$ against the classic/lectin pathway $\mathrm{C} 3$ convertase (known as $\mathrm{C} 4 \mathrm{NeFs}$ ) have also been described [20]. Other autoantibodies include anti-FH, anti-FB, and anti-C3b [31]. However, while the pathogenic role of NeFs in C3G is well established, still there are some uncertainties concerning the best diagnostic methods for their detection, their correlation with the clinical course of the disease, and the most effective therapy to target the production of these autoantibodies [20].

Apart from the aforementioned antibodies, monoclonal gammopathies (MGs) have also been implicated in the pathogenesis of C3G $[11,32]$. Although the underlying mechanisms are not completely understood, monoclonal proteins could act as $\mathrm{NeFs}$ stabilizing the $\mathrm{C} 3$ convertases or act as autoantibodies against complement components [11,32]. Complement hyperactivation in C3G ultimately leads to podocyte dysfunction, as well as mesangial cell injury, and favors the recruitment of inflammatory cells to the glomerular compartment, thereby contributing to progressive kidney damage [33].

\section{Clinical Presentation}

C3G may be diagnosed in both children and adults, although DDD is more frequently diagnosed in children and young adults $[4,10,34]$. When the disease is diagnosed in adults, the presence of an underlying MG must be investigated $[10,11,32,35]$.

The spectrum of clinical manifestations in $\mathrm{C} 3 \mathrm{G}$ is wide, from incidental asymptomatic microscopic hematuria to rapidly progressive forms leading to kidney failure $[9,10,17,36]$. However, the most common presentation in clinical settings is proteinuria with relatively preserved kidney function [10].

Serum C3 hypocomplementemia is a characteristic feature of C3G, although not strictly necessary for the diagnosis $[17,36]$. Patients with $\mathrm{C} 3 \mathrm{NeF}$ tend to have lower serum $\mathrm{C} 3$ levels, as compared to $\mathrm{C} 3 \mathrm{NeF}$-negative patients [20].

The clinical manifestations of $\mathrm{C} 3 \mathrm{G}$ may be preceded by an infection. Hence, a differential diagnosis with postinfectious GN is mandatory. Unfortunately, apart from anti-streptolysin $\mathrm{O}$ and deoxyribonuclease $\mathrm{B}$ antibodies, no other specific markers of the former disease are available, and therefore, the diagnosis may not be straightforward in some cases. An incomplete recovery of kidney 
Fig. 2. Comprehensive characterization of genomic rearrangements in the $\mathrm{CFH}$ gene family: CNVs through MLPA, PCR, WB, and hemolytic assay. CNV, copy number variations; MLPA, multiplex ligation-dependent probe amplification; WB, Western blot.

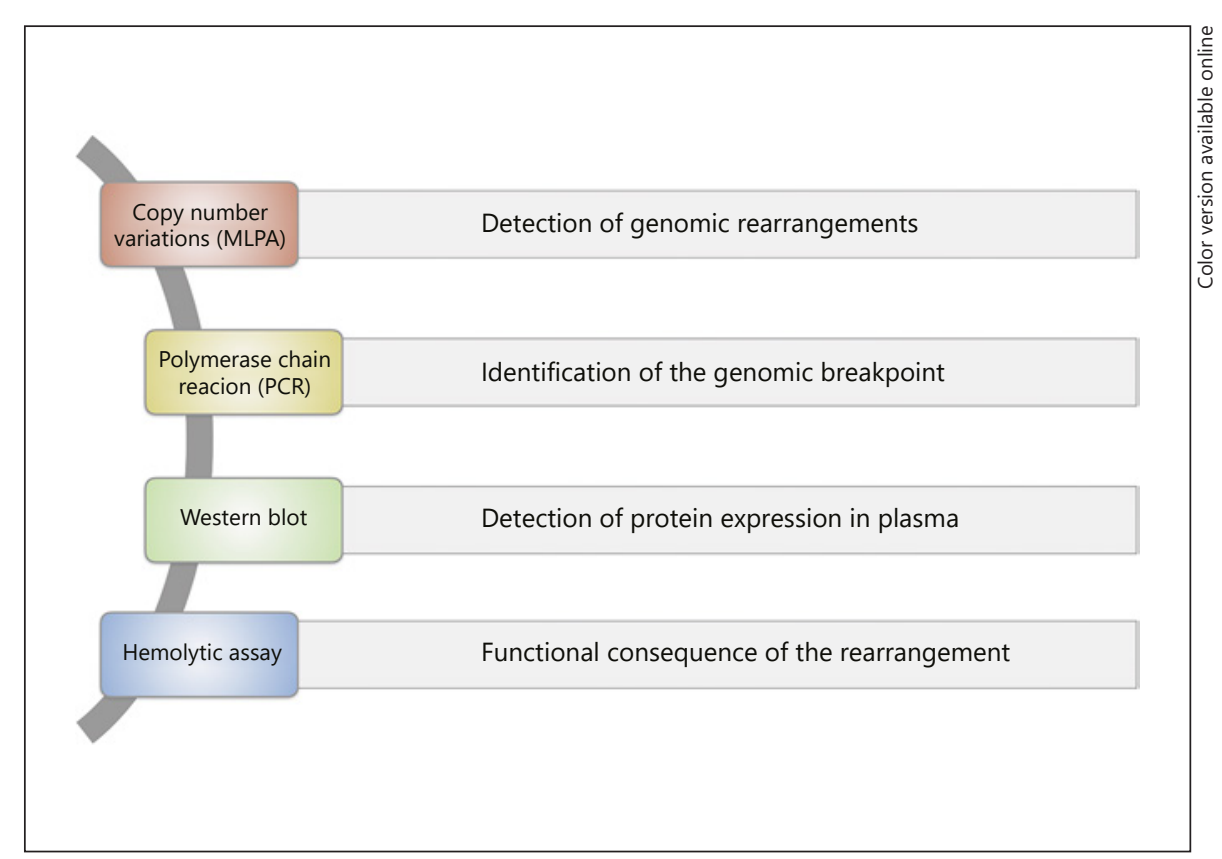

function or the persistence of low serum C3 beyond 8-12 weeks from the initial diagnosis should spark suspicion of C3G [37]. In fact, certain genetic abnormalities in CFHR genes may predispose to developing chronic alternative complement pathway dysregulation [26], and C3G after postinfectious GN.

In addition to renal manifestations, a number of other extrarenal manifestations have also been associated with C3G. For instance, retinal drusen and atrophy have been described in both DDD and C3GN [38]. Although the underlying pathogenesis is poorly understood, it is believed that complement fragments may accumulate in retinal Bruch's membrane following the same mechanism as that of C3G. Thus, patients with C3G should be referred to the ophthalmologist, even if they are asymptomatic, in order to be screened for this condition. However, the clinical course of retinal drusen does not appear to correlate with that of renal disease [38].

Another extrarenal manifestation that may rarely be associated with alternative complement pathway dysregulation is the so-called acquired partial lipodystrophy, which is characterized by a loss of adipose tissue, predominantly affecting the upper half of the body (upper extremities and trunk). This disease is caused by complement-mediated adipocyte lysis triggered by $\mathrm{C} 3 \mathrm{NeF}[20]$.

\section{Histopathologic Characteristics}

The diagnosis of $\mathrm{C} 3 \mathrm{G}$ relies on the histopathologic examination of kidney biopsy specimens, as mentioned earlier. The most common pattern of glomerular injury found under light microscopy is MPGN, accounting for more than $50 \%$ of the cases, followed by mesangial proliferative, diffuse endocapillary proliferative, and diffuse sclerosing GN $[10,14,17,36]$. Cellular and fibrocellular crescents may also be present.

In order to evaluate the prognostic significance of the different histologic lesions found in C3G, a histopathologic index has recently been proposed to assess both the disease activity and chronicity [10]. Seven parameters of activity, including mesangial hypercellularity, endocapillary proliferation, MPGN morphology, leukocyte infiltration, crescent formation, fibrinoid necrosis, and interstitial inflammation, are used. Conversely, 4 parameters of chronicity are scored, comprising global or segmental glomerulosclerosis, tubular atrophy, interstitial fibrosis, and the presence of arteriosclerosis. All components are scored using a semiquantitative scale of $0-3$, except for arteriosclerosis that it is evaluated with a scale of $0-1$. The authors found that both total activity and total chronicity scores were independent predictors of renal survival [10]. While this index has not yet been validated in independent cohorts, it seems reasonable to apply it in clinical 
settings for the prognostic evaluation of patients affected with the disease.

As previously discussed, immunofluorescence staining is the cornerstone technique in the diagnosis of C3G. Bright C3 staining will be found in the mesangium and along the capillary walls, with no significant staining for immunoglobulins, C1q, or light chains [4]. In an attempt to improve its diagnostic accuracy, the potential role of C4d staining has also been studied in C3G for the discrimination of immune-complex-mediated forms [39]. However, while initial studies found that C4d could aid in the diagnosis of the disease, other studies yielded discrepant results [13].

Electron microscopy is necessary to distinguish between the two subtypes, C3GN and DDD [7]. Additional diagnostic techniques include formalin-fixed paraffinembedded processing in cases in which an MG is suspected to be involved in the disease [35], or laser microdissection and mass spectrometry-based proteomics for the identification of alternative complement pathway components [8].

\section{Genetic and Molecular Analysis}

A comprehensive genetic and molecular analysis of complement genes and autoantibodies against complement regulatory factors can be helpful to gain insights into the disease mechanisms (Fig. 2). Complement genes are routinely analyzed by next-generation sequencing. For the identification of common and rare variants associated with the disease, complement gene panels by next-generation sequencing have been developed. However, genomic rearrangements in the $\mathrm{FH}$ gene family cannot be detected with this technique, and thus, a copy number variation analysis, such as multiplex ligation-dependent probe amplification, is necessary. Protein expression can be assessed by Western blot techniques. Conversely, for the detection of specific genomic breakpoints, PCR and DNA sequencing analysis are required. To determine the impact of the mutant proteins in complement regulation, functional assays such as hemolytic assays should be performed.

However, the classification of genetic variants with these techniques and the interpretation of their clinical relevance still remain a challenge, and sometimes a multidisciplinary evaluation of cases is required. One limitation of genome rearrangement analysis is the lack of information regarding its frequency in healthy controls, although due to the high penetrance of the disease, it is assumed that these variants are only found in affected patients.

Update on C3 Glomerulopathy

\section{Natural History and Prognosis}

Spontaneous remissions in C3G are highly unlikely. Nevertheless, extensive clinical experience has shown that in some cases, the disease may remain stable throughout many years despite persistent proteinuria, before patients eventually develop progressive CKD.

According to different case series, almost half of the patients affected with the disease reach end-stage kidney disease over a period of 5 years from clinical diagnosis [ 9 , $10,17]$. However, due to the high clinical variability of the disease, the patterns of kidney function decline may be variable [36].

Initial case series described a significant worse renal survival in patients with DDD as compared to C3GN, although these results have not been confirmed in larger studies [10]. In addition, a study found a predominance of C3GN among Hispanic patients, as opposed to DDD that was more prevalent in Asian patients [10]. On the other hand, there is a broad consensus that older age at diagnosis and higher chronicity in kidney biopsy are independent determinants of worse renal survival $[9,10,17,36]$.

\section{Treatment}

Currently, no targeted therapies are available in clinical settings for the treatment of C3G, although several new molecules are under investigation [40]. The therapeutic alternatives available at present can be classified as supportive therapy, immunosuppression, and anticomplement therapy [41].

\section{Supportive Therapy}

Renin-angiotensin system inhibitors are routinely used due to their renoprotective and anti-proteinuric effects. However, a recent study found that renin had the intrinsic ability to cleave C3 molecules into C3a and C3b, thereby accelerating the alternative complement pathway activation. The use of aliskiren, a direct renin inhibitor, resulted in a significant decrease in complement activation, leading to a lower deposition of $\mathrm{C} 3$ and $\mathrm{C} 5 \mathrm{~b}-9$ in 3 patients with DDD [42].

These results should be treated with caution, although they suggest that renin-angiotensin system inhibitors should not be used in C3G, in order to avoid a consequent increase in renin levels. However, further studies are warranted to better dissect the underlying mechanisms involved and to compare the effect of angiotensin-converting enzyme inhibitors or angiotensin-receptor blockers 
versus aliskiren. At present, there is no solid evidence of the therapeutic benefit of plasma therapy in C3G, even though few case reports have been published, describing both the clearance of $\mathrm{C} 3 \mathrm{NeFs}$ and kidney function improvement $[41,43]$.

\section{Immunosuppression and Other Targeted Therapies}

In the case of conventional immunosuppression, the overall quality of evidence is low, although several observational studies have found a beneficial therapeutic effect of certain immunosuppressive regimens. A nationwide collaborative study coordinated by the Spanish Group for the Study of Glomerular Diseases (GLOSEN) described for the first time the therapeutic effectiveness of corticosteroids plus mycophenolate mofetil (MMF) in 2015 [36]. A well-characterized multicenter cohort including $61 \mathrm{pa}-$ tients with C3GN was recruited, with a regular follow-up that helped analyze the clinical characteristics, response to therapies, and outcomes. Notably, patients who had received any kind of immunosuppression had significant better outcomes than patients who were treated conservatively. However, this favorable effect was more remarkable in those treated with corticosteroids plus MMF [36]. These results were validated by other groups, which also described that higher baseline proteinuria and lower C5b-9 levels were associated with treatment resistance [44].

The main limitation of this study was that complement genetic and molecular analyses were only performed in a small subset of patients. Thus, whether complement genetic abnormalities or the presence of $\mathrm{C} 3 \mathrm{NeFs}$ influences the therapeutic response to this immunosuppressive regimen is yet to be determined.

MMF is a prodrug of mycophenolic acid that blocks purine synthesis in B and T lymphocytes through the inhibition of the enzyme inosine monophosphate dehydrogenase. However, apart from the antiproliferative properties of MMF, several other anti-inflammatory and antifibrotic effects have been described both in vitro and in vivo. Thus, it is tempting to speculate that MMF could reduce inflammation in the glomerular microenvironment in a nonspecific manner and result in improved renal outcomes as compared to other therapies. Whether MMF stabilizes the complement pathway dysregulation in the fluid phase or hampers the production of autoantibodies remains elusive. Therefore, considering the safety and overall good tolerability to corticosteroids plus MMF, perhaps this therapy should be considered as the standard of care in C3G, until newer targeted drugs become available.
One possible therapeutic regimen would be as follows: oral prednisone $0.5-1 \mathrm{mg} / \mathrm{kg} /$ day up to $1-2$ weeks, followed by a slow prednisone tapering over 3-4 months, and MMF 1,000-2,000 mg/day (or mycophenolate sodium 720-1,440 mg/day) according to tolerance or side effects for a period of 1 year or as an indefinite maintenance therapy. Another immunosuppressive agent that has been studied in C3G is rituximab, an anti-CD20 antibody that targets B cells. Likewise, the experience is limited to case reports or small case series. It has been hypothesized that $\mathrm{B}$-cell depletion with rituximab could have a potential therapeutic benefit on $\mathrm{C} 3 \mathrm{NeF}-$ mediated $\mathrm{C} 3 \mathrm{G}$, although the results have not been entirely consistent [20, 41]. Conversely, in cases of C3G in the setting of an MG, a clone-directed approach is the treatment of choice [45].

\section{Anticomplement Therapy}

The successful results achieved with eculizumab in diseases such as paroxysmal nocturnal hemoglobinuria or atypical hemolytic uremic syndrome raised the expectations on its potential use in C3G. Different case studies reported the effectiveness of eculizumab in C3G and ultimately led to the development of a phase I clinical trial including 6 patients ( 3 with DDD and 3 with C3GN, 3 of which had a recurrence of the disease in the transplanted kidney), who received eculizumab for a period of 1 year [46]. At the end of the follow-up, 2 patients showed significant improvement in kidney function, with the additional improvement of mesangial proliferative and endocapillary lesions; 1 patient achieved complete remission with partial reabsorption of electron-dense deposits; and 1 patient had stabilized kidney function with a significant improvement of both mesangial and endocapillary lesions. However, the rest of the cases developed progressive CKD. The overall tolerability was fairly good, with no infectious complications reported. The authors found that patients with higher serum C5b-9 levels had a greater chance to respond to this therapy.

Another recent open-label clinical trial with an off-onoff-on design evaluated the effect of eculizumab on proteinuria in patients with immune-complex-mediated MPGN and C3G, with baseline plasma C5b-9 levels $>1,000 \mathrm{ng} / \mathrm{mL}$ and proteinuria $>3.5 \mathrm{~g} / 24 \mathrm{~h}$ [47]. Only 3 of 10 study patients achieved a significant reduction in $24-\mathrm{h}$ proteinuria, thereby suggesting that other upstream, probably C3-dependent pathways could play a role in the underlying pathogenesis [47].

To summarize, the results of different studies with eculizumab in $\mathrm{C} 3 \mathrm{G}$ revealed that this therapy may only be appropriate in certain cases. Currently, its main clinical 
indication is in those cases with crescentic rapidly progressive disease, although the evidence is still limited and subject to controversy [48]. Currently, there are several ongoing or planned clinical trials using new complement inhibitors targeting early components of the complement cascade, such as factor D (ACH-0144471) and FB (LNP023).

\section{Recurrence in Kidney Transplantation}

Kidney transplantation is a suitable option in $\mathrm{C} 3 \mathrm{G}$, although there is a high risk of recurrence in the kidney allograft. Since the reclassification of MPGN into complement or immune-complex-mediated disease, few case series have been published analyzing this complication. A recent study analyzed the outcomes of 19 patients with C3G (12 C3GN and 7 DDD) who underwent kidney transplantation and found a recurrence rate above $80 \%$, a poor treatment response to different regimens (including rituximab or eculizumab, among others), with the consequent poor allograft survival, particularly in DDD cases [49]. In addition, recurrence may appear earlier and with a more aggressive presentation if associated with an MG [50]. Further studies are warranted to better elucidate the most common genetic abnormalities associated with a higher risk of recurrence, the underlying genotype-phenotype correlations, and the most effective therapeutic strategies in this context.

\section{Conclusions}

In the recent years, several important advances have taken place in the understanding of the pathogenesis of C3G, yet further efforts are needed to bridge current knowledge gaps. Whether a pathogenesis-based classification of the disease improves diagnosis and the identification of high-risk patients, as opposed to the pathologic classification of MPGN, is yet to be confirmed.

Genomic rearrangements, common risk variants, and pathogenic variants in complement genes can be involved in the pathogenesis of $\mathrm{C} 3 \mathrm{G}$. However, due to the high heterogeneity of the disease, in some cases, the genetic contribution may be relevant but not sufficient to explain the onset of the disease. Thus, further studies are needed to better understand genotype-phenotype correlations in C3G.

Renal survival remains unacceptably low, and the risk of recurrence of the disease after kidney transplantation

is a foremost concern. In the next few years, several different complement-targeted therapies will probably become available, but until then, we support the use of corticosteroids plus MMF.

\section{Acknowledgement}

We thank all the members of the Spanish Group for the Study of Glomerular Diseases (GLOSEN) for their valuable contribution to research in glomerular diseases.

\section{Disclosure Statement}

The authors have no conflicts of interest to declare.

\section{Funding Sources}

This study was supported by the Instituto de Salud Carlos III/ Fondo Europeo de Desarrollo Regional (ISCIII/FEDER, grant PI16/01685), Red de Investigación Renal (RedInRen) (RD12/ 0021/0029), and the Autonomous Region of Madrid (S2017/ BMD-3673).

\section{Author Contributions}

All authors contributed equally to this study, revised the paper, and approved the final version of the manuscript.

References

Nephron 2020;144:272-280

DOI: $10.1159 / 000507254$
1 Noris M, Remuzzi G. Overview of complement activation and regulation. Semin Nephrol. 2013;33(6):479-92.

2 de Córdoba SR. Complement genetics and susceptibility to inflammatory disease. Lessons from genotype-phenotype correlations. Immunobiology. 2016;221(6):709-14.

3 Merle NS, Church SE, Fremeaux-Bacchi V, Roumenina LT. Complement system part I: molecular mechanisms of activation and regulation. Front Immunol. 2015;6:262.

4 Bomback AS, Appel GB. Pathogenesis of the C3 glomerulopathies and reclassification of MPGN. Nat Rev Nephrol. 2012;8(11):634-42.

5 Cook HT. Complement and kidney disease. Curr Opin Nephrol Hypertens. 2013;22(3): 295-301.

6 Smith RJH, Appel GB, Blom AM, Cook HT, D'Agati VD, Fakhouri F, et al. C3 glomerulopathy: understanding a rare complementdriven renal disease. Nat Rev Nephrol. 2019; 15:129-43.

7 Cavero T, Praga M. Glomerulopatía C3 : ¿qué sabemos de esta entidad? NefroPlus. 2017;8: 95-107. 
8 Sethi S, Vrana JA, Fervenza FC, Theis JD, Sethi A, Kurtin PJ, et al. Characterization of C3 in C3 glomerulopathy. Nephrol Dial Transpl. 2017;32(3):459-65.

9 Medjeral-Thomas NR, O'Shaughnessy MM, O’Regan JA, Traynor C, Flanagan M, Wong L, et al. C3 glomerulopathy: clinicopathologic features and predictors of outcome. Clin J Am Soc Nephrol. 2014;9(1):46-53.

10 Bomback AS, Santoriello D, Avasare RS, Regunathan-Shenk R, Canetta PA, Ahn W, et al. $\mathrm{C} 3$ glomerulonephritis and dense deposit disease share a similar disease course in a large United States cohort of patients with C3 glomerulopathy. Kidney Int. 2018;93(4):977-85.

11 Ravindran A, Fervenza FC, Smith RJH, Sethi S. C3 glomerulopathy associated with monoclonal Ig is a distinct subtype. Kidney Int. 2018:94(5):1025-2.

12 Hou J, Markowitz GS, Bomback AS, Appel GB, Herlitz LC, Barry Stokes M, et al. Toward a working definition of $\mathrm{C} 3$ glomerulopathy by immunofluorescence. Kidney Int. 2014;85(2): 450-6.

13 Cook HT. Evolving complexity of complement-related diseases: C3 glomerulopathy and atypical haemolytic uremic syndrome. Curr Opin Nephrol Hypertens. 2018;27(3):165-70.

14 Iatropoulos P, Daina E, Curreri M, Piras R, Valoti E, Mele C, et al. Cluster analysis identifies distinct pathogenetic patterns in C3 glomerulopathies/immune complex-mediated membranoproliferative GN. J Am Soc Nephrol. 2018;29:283-94.

15 Iatropoulos P, Noris M, Mele C, Piras R, Valoti E, Bresin E, et al. Complement gene variants determine the risk of immunoglobulin-associated MPGN and C3 glomerulopathy and predict long-term renal outcome. Mol Immunol. 2016;71:131-42.

16 Zhao W, Ding Y, Lu J, Zhang T, Chen D, Zhang $\mathrm{H}$, et al. Genetic analysis of the complement pathway in C3 glomerulopathy. Nephrol Dial Transpl. 2018:33(11):1919, 27.

17 Servais A, Noël LH, Roumenina LT, Le Quintrec M, Ngo S, Dragon-Durey MA, et al. Acquired and genetic complement abnormalities play a critical role in dense deposit disease and other C3 glomerulopathies. Kidney Int. 2012;82(4):454-64.

18 Noris M, Remuzzi G. Genetics of immunemediated glomerular diseases: focus on complement. Semin Nephrol. 2017;37(5):447-63.

19 Zipfel PF, Skerka C, Chen Q, Wiech T, Goodship T, Johnson S, et al. The role of complement in C3 glomerulopathy. Mol Immunol. 2015;67(1):21-30.

20 Corvillo F, Okrój M, Nozal P, Melgosa M, Sánchez-Corral P, López-Trascasa M. Nephritic factors: an overview of classification, diagnostic tools and clinical associations. Front Immunol. 2019;10:886.

21 Donadelli R, Pulieri P, Piras R, Iatropoulos P, Valoti E, Benigni A. Unraveling the molecular mechanisms underlying complement dysregulation by nephritic factors in C3G and ICMPGN. Front Immunol. 2018;9:2329.
22 Ravindran A, Fervenza FC, Smith RJH, De Vriese AS, Sethi S. C3 glomerulopathy: ten years' experience at mayo clinic. Mayo Clin Proc. 2018;93(8):991-1008.

23 Martínez-Barricarte R, Heurich M, ValdesCañedo F, Vazquez-Martul E, Torreira E, Montes T, et al. Human C3 mutation reveals a mechanism of dense deposit disease pathogenesis and provides insights into complement activation and regulation. J Clin Invest. 2010;120(10):3702-12.

24 Lucientes Continente L, Caravaca-Fontán F, Gutiérrez Tenorio J, Márquez Tirado B, Arjona E, García Fernández J, et al. C3 glomerulopathy: an update on complement genetic drivers. Mol Immunol. 2019;114:493.

25 Józsi M, Tortajada A, Uzonyi B, Goicoechea de Jorge E, Rodríguez de Córdoba S. Factor $\mathrm{H}$-related proteins determine complementactivating surfaces. Trends Immunol. 2015; 36(6):374-84

26 Goicoechea de Jorge E, Caesar JJ, Malik TH, Patel M, Colledge M, Johnson S, et al. Dimerization of complement factor H-related proteins modulates complement activation in vivo. Proc Natl Acad Sci USA. 2013;110(12): 4685-90.

27 Gutiérrez Tenorio J, Márquez Tirado B, Merinero $\mathrm{H}$, Tortajada A, Arjona E, Lucientes Continente L, et al. A novel C3G-associated FHR-1 mutant protein provides insight into the molecular basis of complement deregulation. Mol Immunol. 2019;114:487.

28 de Córdoba SR, de Jorge EG. Translational mini-review series on complement factor $\mathrm{H}$ : genetics and disease associations of human complement factor H. Clin Exp Immunol. 2008;151(1):1-13.

29 Goicoechea de Jorge E, López Lera A, BayarriOlmos R, Yebenes H, Lopez-Trascasa M, Rodríguez de Córdoba S. Common and rare genetic variants of complement components in human disease. Mol Immunol. 2018;102:42-57.

30 Marinozzi MC, Chauvet S, Le Quintrec M, Mignotet M, Petitprez F, Legendre C, et al. C5 nephritic factors drive the biological phenotype of C3 glomerulopathies. Kidney Int. 2017;92(5):1232-41.

31 Marinozzi MC, Roumenina LT, Chauvet S, Hertig A, Bertrand D, Olagne J, et al. Antifactor B and anti-C3b autoantibodies in C3 glomerulopathy and ig-associated membranoproliferative GN. J Am Soc Nephrol. 2017; 28(5):1603-13.

32 Zand L, Kattah A, Fervenza FC, Smith RJ, Nasr SH, Zhang Y, et al. C3 glomerulonephritis associated with monoclonal gammopathy: a case series. Am J Kidney Dis. 2013;62(3): 506-14.

33 Takano T, Elimam H, Cybulsky AV. Complement-mediated cellular injury. Semin Nephrol. 2013;33(6):586-01.

34 Riedl M, Thorner P, Licht C. C3 glomerulopathy. Pediatr Nephrol. 2017;32(1):43-57.

35 Caravaca-Fontán F, Gutiérrez E, Delgado Lillo R, Praga M. Gammapatías monoclonales de significado renal. Nefrología. 2017;37:465-77.
36 Rabasco C, Cavero T, Román E, Rojas-Rivera $\mathrm{J}$, Olea T, Espinosa M, et al. Effectiveness of mycophenolate mofetil in $\mathrm{C} 3$ glomerulonephritis. Kidney Int. 2015;88(5):1153-60.

37 Sethi S, Fervenza FC, Zhang Y, Zand L, Meyer $\mathrm{NC}$, Borsa N, et al. Atypical postinfectious glomerulonephritis is associated with abnormalities in the alternative pathway of complement. Kidney Int. 2013;83(2):293-9.

38 Savige J, Amos L, Ierino F, Mack HG, Symons $\mathrm{RC}$, Hughes $\mathrm{P}$, et al. Retinal disease in the $\mathrm{C} 3$ glomerulopathies and the risk of impaired vision. Ophthalmic Genet. 2016;37(4):369-76.

39 Sethi S, Nasr SH, De Vriese AS, Fervenza FC. C4d as a diagnostic tool in proliferative GN. J Am Soc Nephrol. 2015;26(11):2852-9.

40 Ricklin D, Mastellos DC, Reis ES, Lambris JD. The renaissance of complement therapeutics. Nat Rev Nephrol. 2017;14:26-47.

41 Nester CM, Smith RJ. Treatment options for C3 glomerulopathy. Curr Opin Nephrol Hypertens. 2013;22:231-7.

42 Békássy ZD, Kristoffersson AC, Rebetz J, Tati R, Olin AI, Karpman D. Aliskiren inhibits renin-mediated complement activation. Kidney Int. 2018;94:689-700.

43 McCaughan JA, O'Rourke DM, Courtney AE. Recurrent dense deposit disease after renal transplantation: an emerging role for complementary therapies. Am J Transpl. 2012;12(4): 1046-51.

44 Avasare RS, Canetta PA, Bomback AS, Marasa M, Caliskan Y, Ozluk Y, et al. Mycophenolate mofetil in combination with steroids for treatment of $\mathrm{C} 3$ glomerulopathy: a case series. Clin J Am Soc Nephrol. 2018;13(3):40613.

45 Chauvet S, Frémeaux-Bacchi V, Petitprez F, Karras A, Daniel L, Burtey S, et al. Treatment of B-cell disorder improves renal outcome of patients with monoclonal gammopathy-associated C3 glomerulopathy. Blood. 2017; 129(11):1437-47.

46 Bomback AS, Smith RJ, Barile GR, Zhang Y, Heher EC, Herlitz L, et al. Eculizumab for dense deposit disease and C3 glomerulonephritis. Clin J Am Soc Nephrol. 2012;7(5): 748-56.

47 Ruggenenti P, Daina E, Gennarini A, Carrara C, Gamba S, Noris M, et al. C5 convertase blockade in membranoproliferative glomerulonephritis: a single-arm clinical trial. Am J Kidney Dis. 2019;74(2):224-38.

48 Le Quintrec M, Lapeyraque AL, Lionet A, Sellier-Leclerc AL, Delmas Y, Baudouin V, et al. Patterns of clinical response to eculizumab in patients with C3 glomerulopathy. Am J Kidney Dis. 2018;72(1):84-92.

49 Regunathan-Shenk R, Avasare RS, Ahn W, Canetta PA, Cohen DJ, Appel GB, et al. Kidney transplantation in $\mathrm{C} 3$ glomerulopathy: a case series. Am J Kidney Dis. 2018:73(3):316, 23.

50 Zand L, Lorenz EC, Cosio FG, Fervenza FC, Nasr SH, Gandhi MJ, et al. Clinical findings, pathology, and outcomes of C3GN after kidney transplantation. J Am Soc Nephrol. 2014; 25(5):1110-7. 